\title{
Transiente Spannungsmodellierung in umrichtergespeisten Niederspannungsmaschinen mit Steckwicklungen
}

\author{
F. PauliD, Y. Wu, N. Driendl, M. Schröder, K. Hameyer
}

\begin{abstract}
Das Isolierstoffsystem von umrichtergespeisten elektrischen Maschinen ist transienten elektrischen Spannungen ausgesetzt. Diese entstehen aufgrund der endlichen Ausbreitungsgeschwindigkeit der Spannung sowie Induktivitäts- und Kapazitätsbelägen der Wicklung. Durch schnell schaltende Umrichter und den damit verbundenen höheren Spannungsanstiegsgeschwindigkeiten du/dt wird der Einfluss der transienten Spannungen auf das Wicklungssystem weiter erhöht. Eine besondere elektrische Belastung erfährt die Windungsisolierung, welche in Niederspannungsmaschinen häufig als Kupferlackdraht ausgeführt ist. In Steckwicklungen ist die Position jedes Leiters bekannt. Mit einem transienten Modell, das den Spannungsverlauf an jedem Ort der Wicklung abbilden kann, ist es möglich, das Isolierstoffsystem gezielt auszulegen und zu dimensionieren.
\end{abstract}

Schlüsselwörter: elektrische Maschine; Teilentladungen; Isoliersystem; SiC-Umrichter; du/dt

Transient voltage model for inverter-fed low-voltage machines with hairpin windings.

The insulation system of inverter-fed electrical machines is exposed to transient voltages. These are caused by the finite propagation velocity of the voltage as well as by the specific inductance and capacitance of the winding. The influence of transient voltages on the winding system in future drives will be further increased by fast switching inverters and the associated higher voltage slew rates du/dt. In low-voltage machines the winding insulation is often designed as enamelled copper wire. It often experiences the highest electrical stress. In hairpin windings, the position of each conductor is known. A transient model that can map the voltage curve at any location of the winding offers the possiblity to design the insulation system systematically.

Keywords: electrical machine; partial discharge; insulation system; SiC-inverter; du/dt

Eingegangen am 9. Dezember 2019, angenommen am 22. April 2020, online publiziert am 19. Mai 2020 (C) The Author(s) 2020

\section{Einleitung}

Durch die Elektrifizierung des Individualverkehrs werden große Stückzahlen elektrischer Niederspannungsmaschinen benötigt. Eine wirtschaftliche Fertigung kann durch einen hohen Automatisierungsgrad gewährleistet werden. Dieser kann bei Maschinen mit Steckwicklungen (oder Hairpin-Wicklungen) realisiert werden. Um Produktionskosten zu senken, wird dabei eine Verbesserung bestehender Fertigungsprozesse angestrebt [1, 2]. Um schnell ladende Fahrzeuge mit hohen Leistungen realisieren zu können, werden hohe Zwischenkreisspannungen benötigt. Gleichzeitig werden vermehrt Umrichter mit hohen Spannungsanstiegsgeschwindigkeiten $\mathrm{d} u / \mathrm{d} t$ eingesetzt, was zu ausgeprägten Überschwingvorgängen führen kann. Beides stellt eine besondere Belastung des Isolierstoffsystems dar und kann zu Teilentladungen und damit zu einer drastischen Verkürzung der Lebensdauer des Wicklungssystems führen [3]. Im Gegensatz zu Statoren mit einer verteilten Runddrahtwicklung, ist bei Statoren mit Steckwicklungen die Position jedes einzelnen Leiters in der Nut bekannt. Dies ermöglicht eine gezielte Auslegung der Windungsisolierung, indem die Isolierschichtdicken an die Spannung zwischen zwei benachbarten Leitern angepasst werden. Diese Spannung ist allerdings nicht an jeder Position des Stators messtechnisch zugänglich. Aus diesem Grund werden transiente Modelle der Wicklung benötigt. Das Verhalten elektri- scher Leitungen kann durch die Telegraphengleichung beschrieben werden. Diese lässt sich ebenfalls durch eine Verkettung unendlich vieler Ersatzschaltungen ausdrücken (vgl. Abb. 1). In der Wicklung einer elektrischen Maschine treten neben nichtlinearem Materialverhalten auch Kopplungseffekte auf, die durch die Telegraphengleichung nicht erfasst werden. Eine Möglichkeit, das transiente Verhalten der Wicklung zu modellieren, ist es, modifizierte verkettete Ersatzschaltbilder zu nutzen, die Nichtlinearitäten und Kopplungen in einem bestimmten Frequenzbereich abbilden können $[4,5,10]$. Die konzentrierten Parameter der Modelle lassen sich entweder durch Messungen oder durch elektromagnetische Finite-Elemente-Simulationen (FES) bestimmen [6, 7]. Im Rahmen dieses Artikels wird ein Hairpin-Stator mit der in [8] vorgestellten Methode modelliert. Ein weiteres transientes Wicklungmodell ist

Pauli, Florian, Institut für Elektrische Maschinen (IEM), RWTH Aachen University, Schinkelstraße 4, 52062 Aachen, Deutschland (E-Mail: florian.pauli@iem.rwth-aachen.de); Wu, Yixuan, Institut für Elektrische Maschinen (IEM), RWTH Aachen University, Schinkelstraße 4, 52062 Aachen, Deutschland; Driendl, Niklas, Institut für Elektrische Maschinen (IEM), RWTH Aachen University, Schinkelstraße 4, 52062 Aachen, Deutschland; Schröder, Michael, Institut für Elektrische Maschinen (IEM), RWTH Aachen University, Schinkelstraße 4, 52062 Aachen, Deutschland; Hameyer, Kay, Institut für Elektrische Maschinen (IEM), RWTH Aachen University, Schinkelstraße 4, 52062 Aachen, Deutschland 


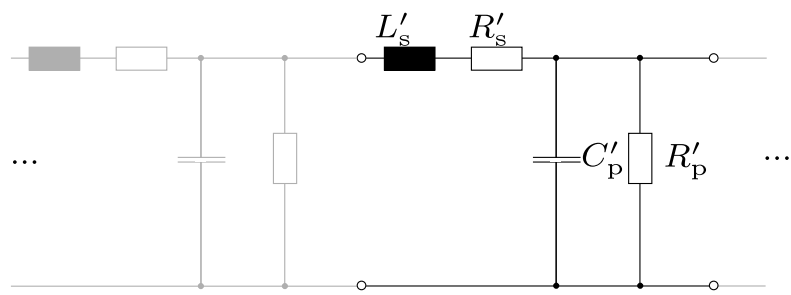

Abb. 1. Ersatzschaltbild zur Modellierung des Hochfrequenzverhaltens einer Leitung

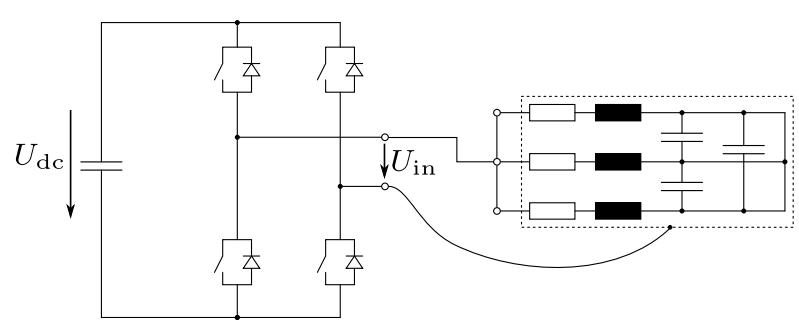

Abb. 2. Verschaltung des Stators

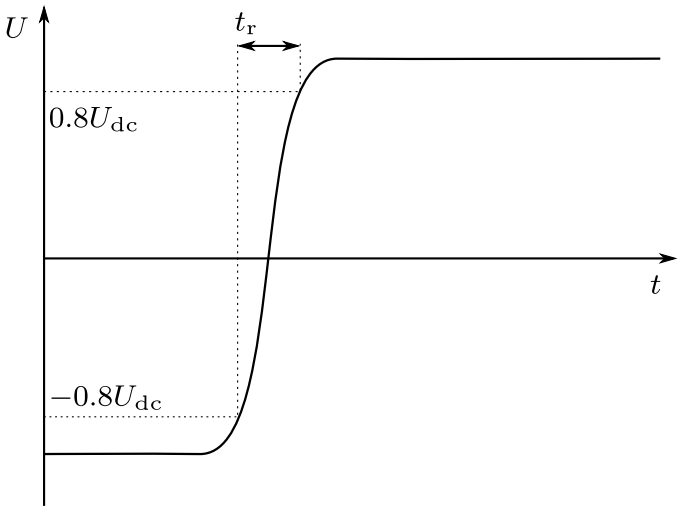

Abb. 3. Idealisierter Spannungsanstieg

durch die Lösung der Telegraphengleichung im Frequenzbereich gegeben.

\section{Versuchsaufbau}

In dieser Veröffentlichung wird die Ausbreitung der Spannung anhand der Statorwicklung eines Lenkmotors untersucht. Die Wicklung des Stators wird dabei dreisträngig kurzgeschlossen und mit einer H-Brücke verbunden (vgl. Abb. 2). Es befindet sich kein Rotor innerhalb der Statorbohrung.

Die Schaltung versorgt den Prüfling mit einer bipolaren Pulsspannung. Ein idealisierter Spannungsanstieg ist in Abb. 3 dargestellt. Die Spannungsanstiegszeit $t_{r}$ bezeichnet dabei die Spanne zwischen den Zeitpunkten, an denen $10 \%$ und $90 \%$ des Spannungsanstieges abgeschlossen sind. Bei der hier vorgenommenen Modellierung wird der idealisierte Spannungsanstieg durch die Fehlerfunktion beschrieben.

Bedingt durch Induktivitäts- und Kapazitätsbeläge der Wicklung sowie der Leitungen provoziert dabei jeder Schaltvorgang ein Überschwingen. Mit Hilfe eines Oszilloskops mit mehren Kanälen kann der Spannungsverlauf zwischen den Eingangsklemmen und dem geerdeten Stator als Referenzsignal gemessen werden. Mit einem

\section{Tab. 1. Kapazitäten des Whitebox-Modells}

\begin{tabular}{ll}
\hline Parameter & \\
\hline Außendurchmesser & $85 \mathrm{~mm}$ \\
Innendurchmesser & $51 \mathrm{~mm}$ \\
Aktive Länge & $75 \mathrm{~mm}$ \\
Polpaarzahl & 2 \\
Anzahl Statorzähne & 36 \\
Lochzahl & 3 \\
\hline
\end{tabular}

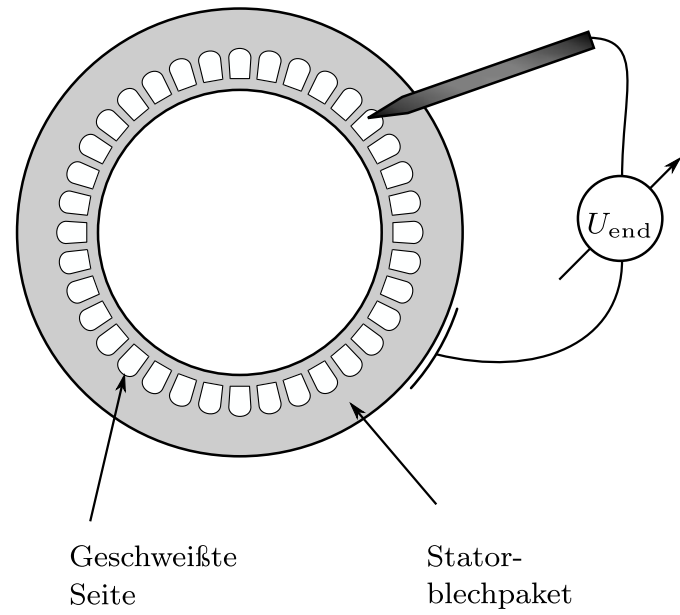

(a) Schematische Darstellung des Messaufbaus.

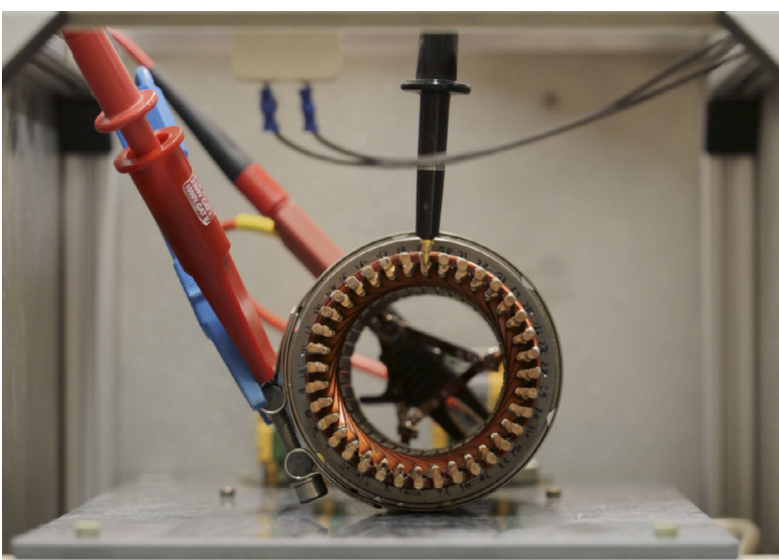

(b) Foto des Messaufbaus.

\section{Abb. 4. Messaufbau}

zweiten Messkanal wird die Spannung zwischen jedem der Schweißpunkte der Wicklung und dem geerdeten Stator gemessen. Mit Hilfe des Referenzsignals kann dann die Spannung zwischen zwei beliebigen Leitern durch Subtraktion berechnet werden. Der Messaufbau ist in Abb. 4 gegeben. Die Zwischenkreisspannung bei den hier durchgeführten Versuchen beträgt $U_{\mathrm{dc}}=20 \mathrm{~V}$. Die Daten des Stators können Tab. 1 entnommen werden.

Bei der Modellbildung wird der Spannungsverlauf entlang der Wicklung betrachtet. Der im Folgenden verwendete Ausdruck Windungsnummer bezeichnet dementsprechend die elektrische Position der betrachteten Windung. Eine Aussage darüber, in welchen Nuten die entsprechende Windung platziert ist, ist mit Hilfe des in Abb. 5 gegebenen Wickelschemas möglich. Da die Wicklung symmetrisch 


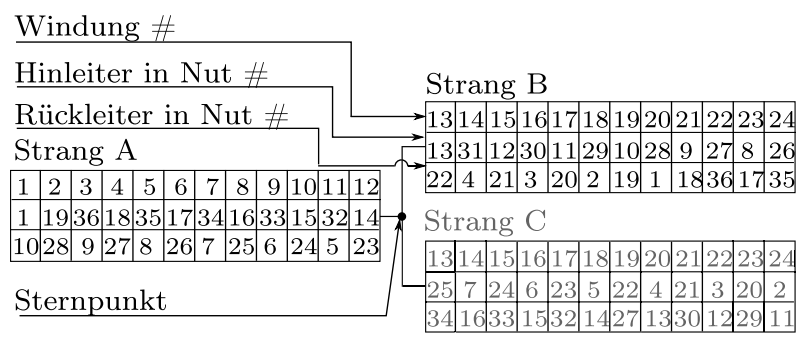

Abb. 5. Windungsnummern (obere Zeile) und zugehörige Nutpositionen (untere zwei Zeilen)

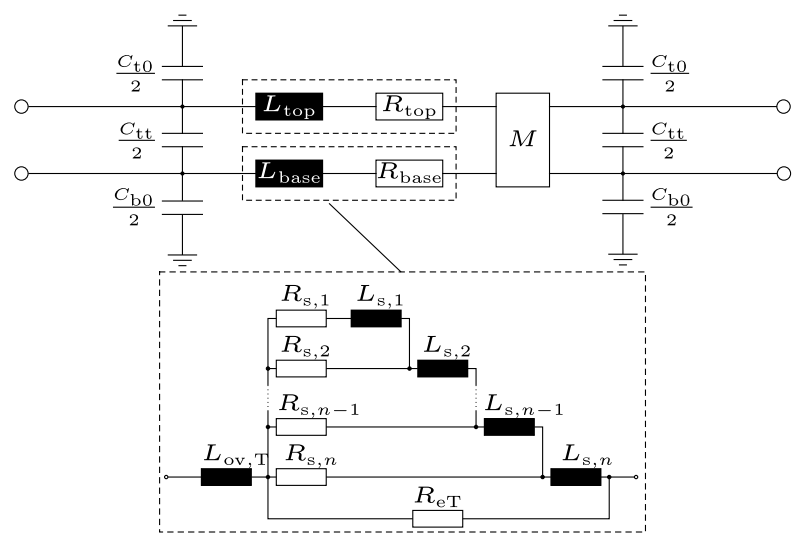

(a) Ersatzschaltbild für eine Nut.

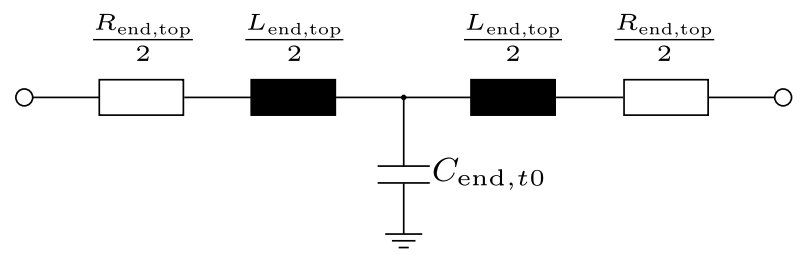

(b) Ersatzschaltbild eines Leiterabschnitts im Wickelkopf.

Abb. 6. Ersatzschaltbilder zur Modellierung des Hochfrequenzverhaltens der Wicklung

ist und immer der Spannungsverlauf von den Klemmen von Strang A zu den Klemmen von Strang B betrachtet wird, ist Strang $C$ in der Abbildung ausgegraut.

\section{Modellierung}

In diesem Abschnitt werden zwei verschiedene Modelle zur Berechnung der transienten Spannung vorgestellt. Das Lumped-ParameterModell wird dabei ausschließlich mit Hilfe der Daten aus einer FE-Simulation parametriert und beschreibt darum ein WhiteboxModell. Bei der Anwendung der Telegraphengleichung im Frequenzbereich werden sowohl Messdaten, als auch Geometriedaten der Wicklung verwendet. Darum handelt es sich hierbei um eine Greybox-Modell.

\subsection{Whitebox-Modell}

Das Ersatzschaltbild, mit welchem das transiente Verhalten der Maschine abgebildet wird, besteht aus zwei verschiedenen Gruppen. Eine Gruppe stellt dabei den Wickelkopf dar, die andere den Teil der Wicklung, welcher in der Nut verläuft. Die hier modellierte Maschine beinhaltet pro Nut zwei Leiter, die aufgrund der Sehnung
Tab. 2. Kapazitäten des Whitebox-Modells

\begin{tabular}{lc}
\hline Parameter & Kapazität in $\mathrm{pF}$ \\
\hline$C_{\mathrm{t0}}$ & 49.01 \\
$C_{\mathrm{bo}}$ & 58.63 \\
$C_{\mathrm{tt}}$ & 41.55 \\
$C_{\text {end,to }}$ & 1.80 \\
$C_{\text {end,bo }}$ & 1.59 \\
$C_{\text {end,tt,top }}$ & 1.32 \\
$C_{\text {end,t,base }}$ & 1.42 \\
\hline
\end{tabular}

immer zu zwei verschiedenen Strängen gehören. Ströme und Spannungen innerhalb der Leiter werden durch kapazitive und induktive Kopplung voneinander beeinflusst. Dies wird im Ersatzschaltbild durch die Kapazität $C_{\mathrm{tt}}$ und die Koppelinduktivität $M$ berücksichtigt (siehe Abb. 6(a)). Um die Frequenzabhängigkeit von Widerstand und Induktivitäten abzubilden, wird eine sogenannte $L R$-Ladder verwendet. Der Wickelkopf wird durch das in Abb. 6(b) dargestellte Ersatzschaltbild modelliert.

In Abb. 7(a) ist die Leiteranordnung in zwei benachbarten Nuten schematisch dargestellt. Der helle Leiter liegt dabei im Nutgrund, der dunkle an der Nutöffnung. Da die Leiter aus der jeweiligen Lage im Wickelkopf direkt nebeneinander verlaufen, ist auch hier eine kapazitive Kopplung vorhanden. Dies wird in der in Abb. 7(b) abgebildeten Verschaltung von Nuten und Wickelköpfen zum gesamten Statormodell deutlich.

Aus numerischen Gründen wird mit Hilfe einer Stern-PolygonTransformation (siehe [9]) die kapazitive Kopplung zwischen benachbarten Leitern im Wickelkopf auf den geerdeten Stator bezogen und zur Kapazität $C_{\text {end,o }}$ hinzuaddiert. Die Parametrierung des Modells erfolgt mit Hilfe einer elektrostatischen und einer magnetischen FESimulation. Mit der elektrostatischen Simulation werden die kapazitiven Kopplungen in der Nut und im Wickelkopf erfasst. Für die betrachtete Maschine ergeben sich die numerischen Werte aus Tab. 2.

Die magnetische FE-Simulation wird zur Parametrierung der $L R$ Ladder verwendet. Dazu wird die Simulation bei 40 verschiedenen, logarithmisch verteilten Frequenzen im Bereich von $f=1 \mathrm{kHz}$ bis $f=100 \mathrm{MHz}$ durchgeführt. Mit Hilfe eines Parameterfittings werden Induktivitäten und Kapazitäten einer LR-Ladder mit vier Elementen so angepasst, dass sie den Impedanzverlauf der Simulation wiedergeben kann. In Abb. 8 sind simulierte und durch die LR-Ladder beschriebenen Impedanzen aufgetragen.

Die identifizierten Parameter sind in Tab. 3 aufgelistet.

\subsection{Greybox-Modell}

Die Modellierung der zeit- und ortsabhängigen Spannungen $u(x, t)$ und Ströme $i(x, t)$ im Frequenzbereich basiert auf der Telegraphen- 


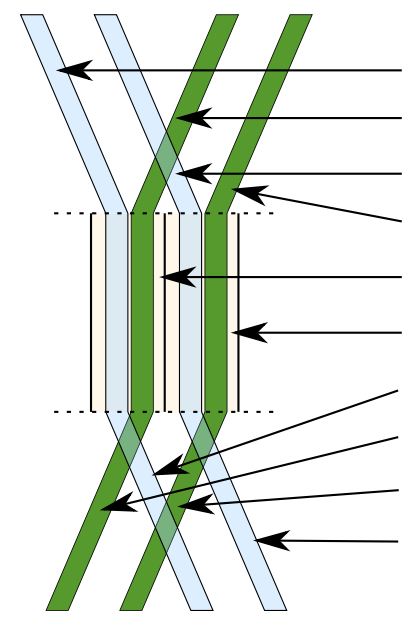

End $_{\text {top,gebogen, } n}$

End $_{\text {base,gebogen, } n}$

End $_{\text {top }, \text { gebogen }, n+1}$

End $_{\text {base,gebogen, } n+1}$

Nut $n$

Nut $n+1$

End $_{\text {top, geschweißt, } n}$

End $_{\text {base,geschweißt, } n}$

End $_{\text {base, geschweißt, } n+1}$

End $_{\text {top,geschweißt, } n+1}$

(a) Anordnung der Leiter in benachbarten Nuten.

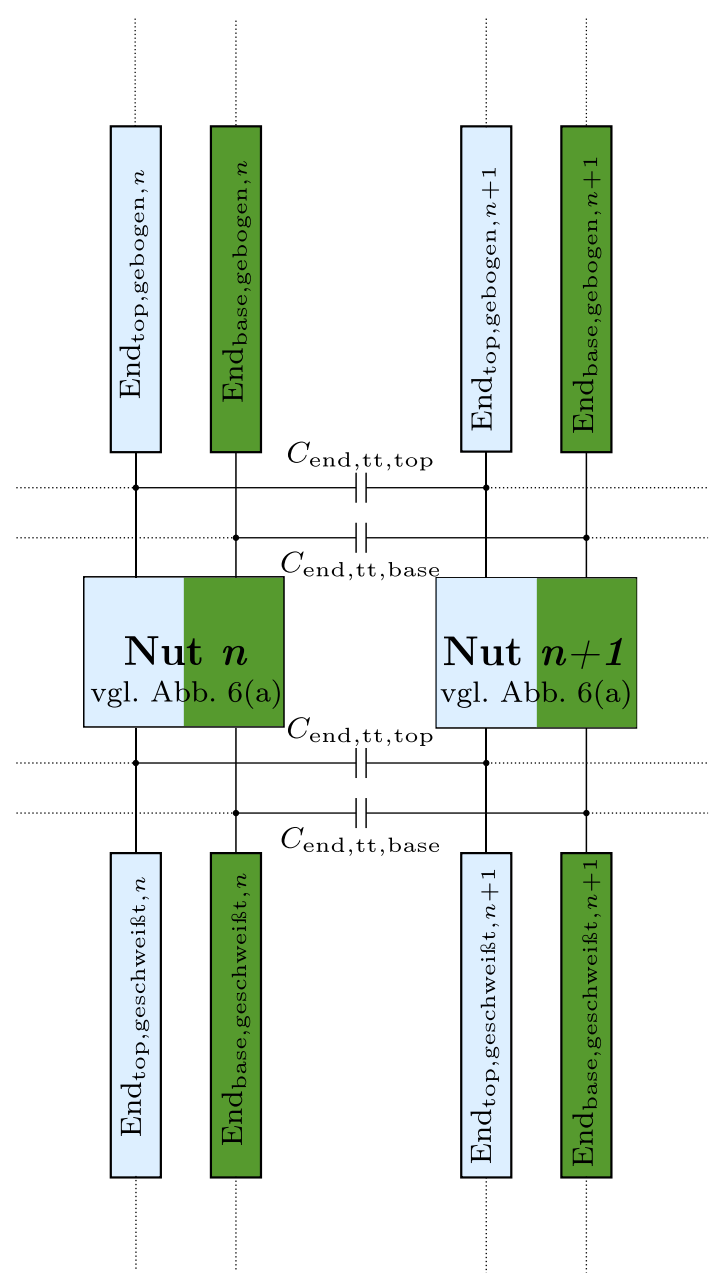

(b) Verschaltung der einzelnen Ersatzschaltbilder zur Modellierung des gesamten Stators.

Abb. 7. Ersatzschaltbilder zur Modellierung des Hochfrequenzverhaltens der Wicklung

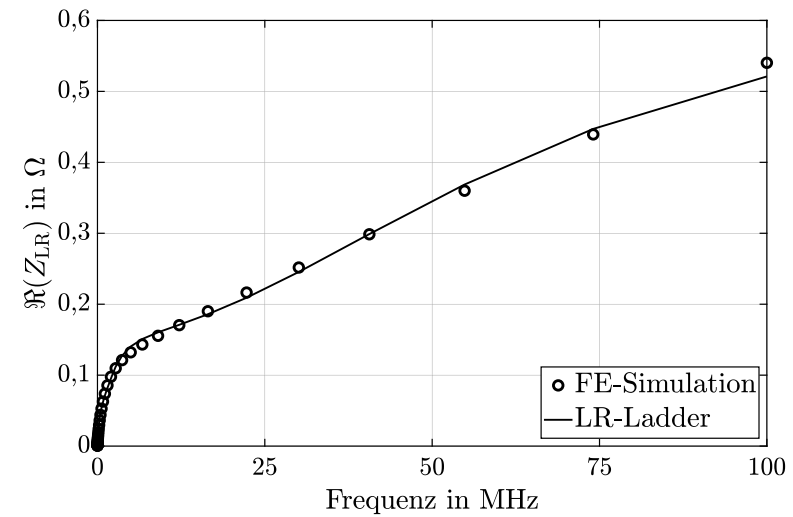

(a) Durch FE-Simulation und durch LR-Ladder wiedergegebener Realteil der Impedanz.

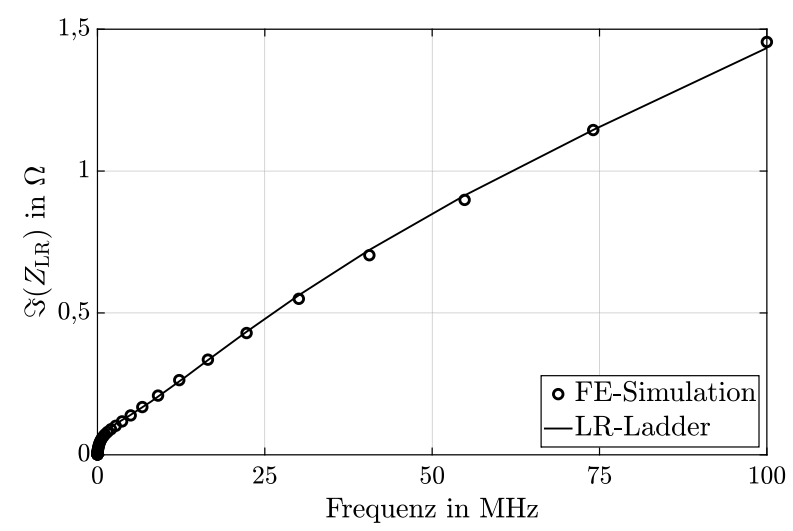

(b) Durch FE-Simulation und durch LR-Ladder wiedergegebener Imaginärteil der Impedanz.

Abb. 8. Durch FE-Simulation und durch LR-Ladder wiedergegebener (a) Realteil und (b) Imaginärteil der Impedanz

Tab. 3. Parameter der LR-Ladder

\begin{tabular}{lcll}
\hline Parameter & Widerstand in $\mu \Omega$ & Parameter & Induktivität in $\mu \mathrm{H}$ \\
\hline$R_{1}$ & 4.09 & $L_{1}$ & 34.89 \\
$R_{2}$ & 65.64 & $L_{2}$ & 14.41 \\
$R_{3}$ & 192.62 & $L_{3}$ & 2.10 \\
$R_{4}$ & 684.75 & $L_{4}$ & 1.89 \\
\hline
\end{tabular}

gleichung:

$$
\begin{aligned}
\frac{\partial}{\partial x} u(x, t) & =-L^{\prime} \frac{\partial}{\partial t} i(x, t)-R^{\prime} i(x, t) \\
\frac{\partial}{\partial x} i(x, t) & =-C^{\prime} \frac{\partial}{\partial t} u(x, t)-G^{\prime} u(x, t) .
\end{aligned}
$$

Dabei beschreiben $L^{\prime}$ und $C^{\prime}$ Induktivitäts- und Kapazitätsbeläge und $R^{\prime}$ und $G^{\prime}$ Widerstands- und Leitwertbeläge. Die Zeitabhängigkeit in der Telegraphengleichung wird dabei eliminiert, indem rein sinusförmige Spannungen betrachtet werden. Gleichung (1) kann dann entsprechend zu

$$
\begin{aligned}
& \underline{U}(x)=\underline{U}_{h 0} \mathrm{e}^{-\underline{\gamma x}}+\underline{U}_{\mathrm{r} 0} \mathrm{e}^{+\underline{\gamma x}} \\
& \underline{I}(x)=\frac{\underline{U}_{\mathrm{h} 0}}{\underline{Z}} \mathrm{e}^{-\underline{\gamma x}}-\frac{\underline{U}_{\mathrm{r} 0}}{\underline{Z}} \mathrm{e}^{+\underline{\gamma x}}
\end{aligned}
$$




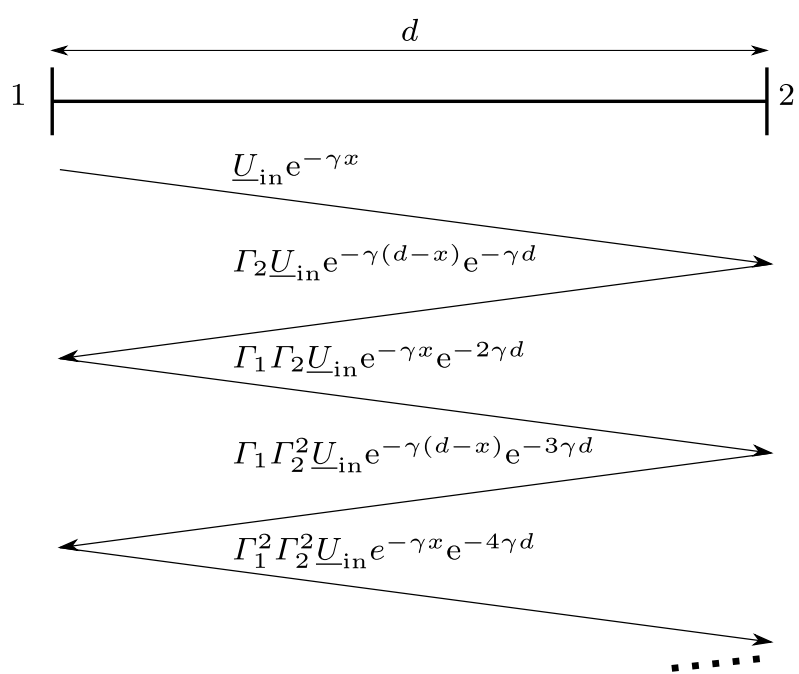

Abb. 9. Schematische Darstellung des Reflexionsvorganges an einer einfachen Leitung

vereinfacht werden. Dabei ist $\underline{Z}$ der komplexe Wellenwiderstand und $\underline{\gamma}$ die komplexe Ausbreitungskonstante

$$
\underline{\gamma}=\alpha+\mathrm{j} \beta .
$$

$\alpha$ beschreibt dabei den dissipativen Anteil der Ausbreitungskonstante und $\beta$ die Phasenverschiebung. $\alpha$ und $\beta$ werden dabei von den Leitungsparametern bestimmt:

$$
\begin{aligned}
& \alpha^{2}=\left(R^{\prime} G^{\prime}-\omega^{2} L^{\prime} C^{\prime}\right)+\left(\omega L^{\prime} G^{\prime}-\omega C^{\prime} R^{\prime}\right)^{2}, \\
& \arctan (\beta)=\frac{\omega L^{\prime} G^{\prime}+\omega C^{\prime} R^{\prime}}{R^{\prime} G^{\prime}-\omega^{2} L^{\prime} C^{\prime}} .
\end{aligned}
$$

Mit Hilfe einer Fourier-Transformation kann die dominante Frequenz, welche durch den Spannungssprung an den Eingangsklemmen angeregt wird, identifiziert werden. Die Ausbreitungskonstante lässt sich durch Messung des Eingangssignals und eines weiteren Signals bestimmen.

Wird eine Welle betrachtet, die von den Klemmen eines Stranges zu den Klemmen der anderen beiden Stränge läuft, passiert diese den Sternpunkt. An dieser Stelle halbiert sich die Impedanz der Wicklung, was zu einem Reflexionsvorgang führt. Für den Transmissionskoeffizienten $\underline{I}_{S P}$ und den Reflexionskoeffizienten $\underline{\Gamma}_{S P}$ gilt am Sternpunkt

$$
\underline{T}_{\mathrm{SP}}=-2 \cdot \underline{\Gamma}_{\mathrm{SP}} .
$$

Wird jedoch dieselbe Eingangsspannung gleichzeitig auf alle drei Stränge gegeben, muss die Diskontinuität am Sternpunkt aufgrund der Symmetrie nicht betrachtet werden, da sich die Spannungen aller drei Stränge überlagern. Der Refelexionsvorgang an einer Leitung, bei der einseitig eine Spannungswelle einläuft, ist schematisch in Abb. 9 dargestellt. Der Eingang der Leitung ist als Klemme 1 gekennzeichnet. Die Welle wird mit der Ausbreitungskonstante $\gamma$ gedämpft und an der Klemme 2 mit dem Reflexionskoeffizienten $\underline{\Gamma}_{2}$ reflektiert. Daraufhin läuft die Welle zurück zur Klemme 1, wo sie wiederum mit dem Reflexionskoeffizienten $\underline{\Gamma}_{1}$ reflektiert wird. Die hin- und rücklaufenden Wellen überlagern sich dabei. Die resultie-

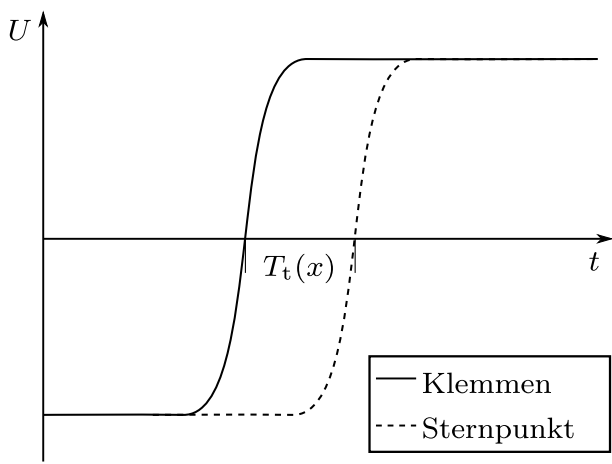

Abb. 10. Totzeit zwischen Spannungssprung an den Eingangsklemmen und am Sternpunkt

rende Spannung an der Position $x$ lässt sich wie folgt darstellen:

$$
\begin{aligned}
& \underline{U}_{\text {singleLine }}(x)= \\
& =\underline{U}_{\text {in }}\left(\mathrm{e}^{-\underline{\gamma x}}+\mathrm{e}^{-\underline{\gamma}(d-x)} \mathrm{e}^{-\underline{\gamma d}} \underline{\Gamma}_{2}\right) \cdot \sum_{k=0}^{\infty}\left(\underline{\Gamma}_{1} \underline{\Gamma}_{2} \mathrm{e}^{-2 \underline{\gamma} d}\right)^{k}= \\
& =\underline{U}_{\text {in }} \frac{\mathrm{e}^{-\underline{\gamma x}}+\mathrm{e}^{-\underline{\gamma d} d} \mathrm{e}^{-\underline{\gamma}(d-x)} \underline{\Gamma}_{2}}{1-\underline{\Gamma}_{1} \underline{\Gamma}_{2} \mathrm{e}^{-2 \underline{\gamma} d}} .
\end{aligned}
$$

Bei dem verwendeten Messaufbau sind alle drei Klemmen des Stators mit demselben Potential verbunden. Darum muss eine beidseitig einlaufende Spannungswelle betrachtet werden. Die Reflexionskoeffizienten sind an allen Klemmen gleich. Darum gilt:

$$
\underline{\Gamma}_{1}=\underline{\Gamma}_{2}=\underline{\Gamma}_{\mathrm{t}} .
$$

Unter dieser Voraussetzung gilt für die Spannung $\underline{U}$ an der Position $x$ :

$$
\underline{U}(x)=\underline{U}_{\text {in }} \frac{\mathrm{e}^{-\underline{\gamma x} x}+\mathrm{e}^{-\underline{\gamma}(d-x)}+\underline{\Gamma}_{\mathrm{t}} \mathrm{e}^{-\underline{\gamma d} d}\left(\mathrm{e}^{\mathrm{e}^{\gamma x}}+\mathrm{e}^{\underline{\gamma}(d-x)}\right)}{1-\underline{\Gamma}_{\mathrm{t}}^{2} \mathrm{e}^{-2 \underline{\gamma} d}} .
$$

Im Allgemeinen sind Ausbreitungkonstante $\underline{\gamma}$ und Reflexionskonstante $\underline{\Gamma_{\mathrm{t}}}$ frequenzabhängig. Um mit Hilfe der Wellentheorie die Spannungsverteilung in einem Stator betrachten zu können, wird die Modellierung der Spannungsverteilung zunächst auf eine dominante Frequenz reduziert. Hierzu muss die Spannung zwischen den Eingangsklemmen und dem geerdeten Stator, sowie zwischen Sternpunkt und Stator gemessen werden. Von der gemessenen Spannung wird dann ein idealer Spannungspuls subtrahiert. Da die Ausbreitungsgeschwindigkeit der Spannung endlich ist, muss eine Totzeit zwischen Klemmen und Sternpunkt berücksichtigt werden (vgl. Abb. 10).

Das resultierende Signal wird Fourier-transformiert. In Abb. 11 ist zu erkennen, dass die Maxima der Amplituden aller Windungen in einem schmalen Frequenzband liegen. Im Folgenden wird die Frequenz, an welcher das Maximum auftritt, als dominante Frequenz bezeichnet. Mit Hilfe der Messdaten von Eingangsklemmen und Sternpunkt, kann nun Gl. (9) parametriert werden. Dabei gilt an den Klemmen:

$$
\underline{U}(0)=\underline{U}(d)=\underline{U}_{\text {in }} \frac{1+\mathrm{e}^{-\underline{\gamma} d}}{1-\mathrm{e}^{-\underline{\gamma} d} \underline{\Gamma}_{\mathrm{t}}} .
$$

Die Spannung am Sternpunkt beträgt:

$$
\underline{U}(d / 2)=\underline{U}_{\text {in }} \frac{2 \mathrm{e}^{-\underline{\gamma} d / 2}}{1-\mathrm{e}^{-\underline{\gamma} d} \underline{\Gamma}_{\mathrm{t}}} .
$$




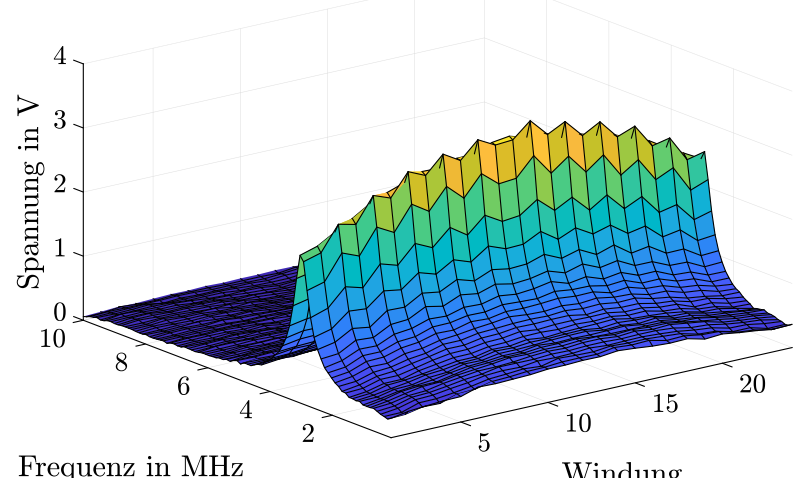

Frequenz in $\mathrm{MHz}$

Windung

Abb. 11. Spektrum der Spannung in jeder Nut nach Subtraktion eines idealisierten Sprunges

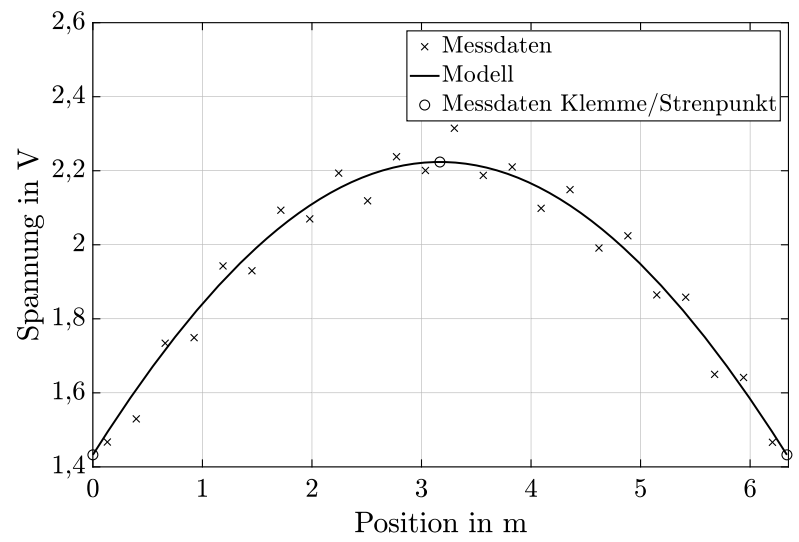

Abb. 12. Gemessene und berechnete Amplitude

In Abb. 12 werden die gemessene und die mit Gl. (9) bestimmte Amplitude miteinander verglichen. Die Stützpunkte zur Parametrierung der Gleichung (Sternpunkt und Klemmen) sind mit einem Kreis gekennzeichnet.

Die Modellierung der Spannung basiert ausschließlich auf den Messdaten von Sternpunkt und Maschinenklemmen. Zunächst wird ein idealisierter Sprung von den Messdaten abgezogen. Das verbleibende Signal wird Fourier-transformiert und die dominante Frequenz wird identifiziert. Für diese Frequenz wird Gl. (9) parametriert, mit deren Hilfe nun für die dominante Kreisfrequenz $\omega^{*}$ Amplitude und Phasenverschiebung der Spannung an jeder Position der Wicklung berechnet werden können. Der Spannungsverlauf für eine festgelegte Position wird bestimmt, indem zunächst die komplexen Faktoren $\underline{k}_{1}$ und $\underline{k}_{2}$ berechnet werden. Diese entsprechen den Quotienten aus der Spannung an der betrachteten Position $\underline{U}\left(x^{*}, j \omega^{*}\right)$ und der Sternpunktspannung $\underline{U}\left(d / 2, j \omega{ }^{*}\right)$ beziehungsweise der Klemmenspannung $\underline{U}\left(0, j \omega^{*}\right)=\underline{U}\left(d, j \omega^{*}\right)$ :

$$
\begin{aligned}
& \underline{k}_{1}=\frac{\underline{U}\left(x^{*}, j \omega^{*}\right)}{\underline{U}\left(d / 2, j \omega^{*}\right)}, \\
& \underline{k}_{2}=\frac{\underline{U}\left(x^{*}, j \omega^{*}\right)}{\underline{U}\left(0, j \omega^{*}\right)} .
\end{aligned}
$$

Anschließend werden die Spektren aus der Messung an den Klemmen und am Sternpunkt abhängig von ihrer Postion $x^{*}$ mit den Fak-

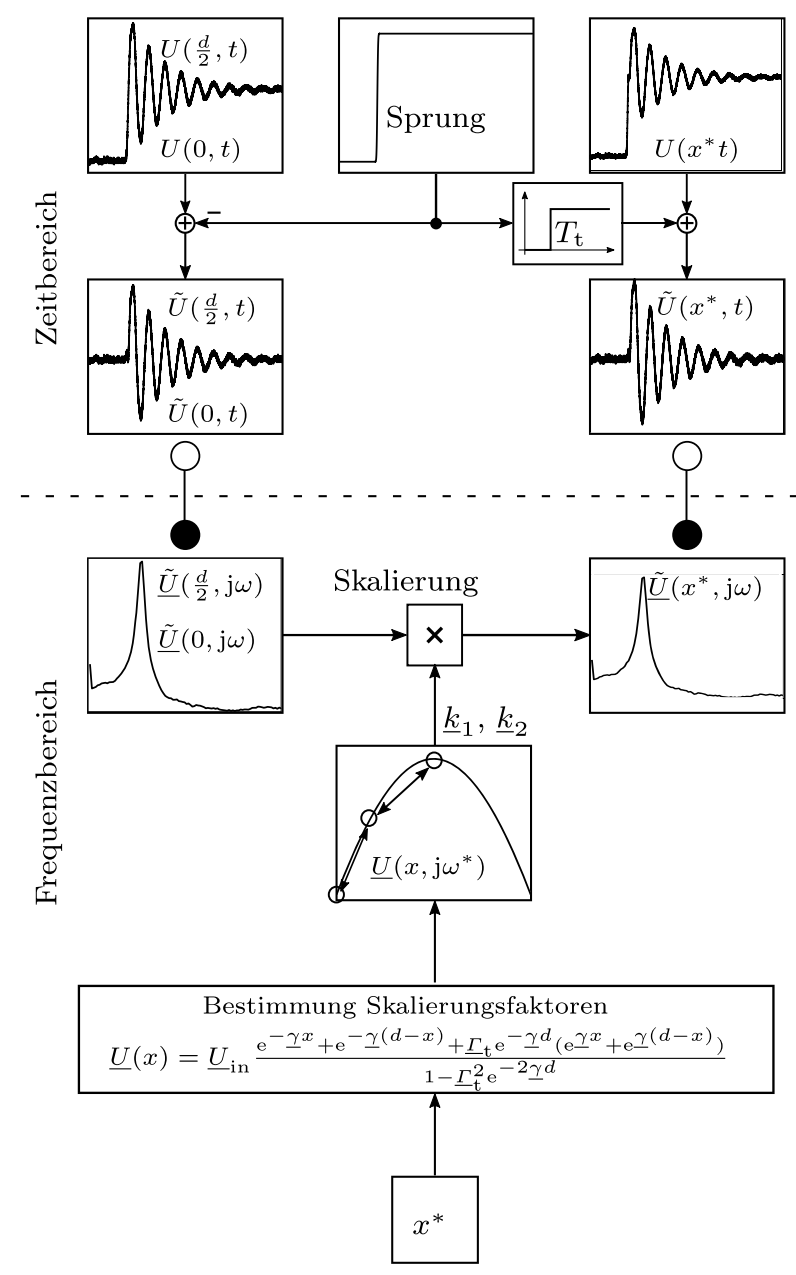

Abb. 13. Verfahren zur Anwendung des Greybox-Modells

toren $\underline{k}_{1}$ beziehungsweise $\underline{k}_{2}$ skaliert:

$$
\begin{aligned}
\underline{U}\left(x^{*}, j \omega\right)= & \left(1-\left|\frac{x^{*}-d / 2}{d / 2}\right|\right) \underline{k}_{1} \underline{U}(d / 2, j \omega) \\
& +\left|\frac{x^{*}-d / 2}{d / 2}\right| \underline{k}_{2} \underline{U}(0, j \omega) .
\end{aligned}
$$

Der Zeitverlauf der Spannung ist durch inverse Fourier-Transformation und Addition des idealisierten Spannungssprungs gegeben. Das Verfahren wird durch Abb. 13 veranschaulicht. Die Bezeichnungen der verwendeten Spannungen sind durch Tab. 4 gegeben.

\section{Mess- und Simulationsergebnisse}

In diesem Kapitel werden die Simulationsergebnisse, die mit den zuvor vorgestellten Methoden erzielt werden, vorgestellt und mit den Messergebnissen verglichen.

\subsection{Lumped-Parameter-Modell}

In Abb. 14 ist der zeitliche Verlauf der gemessenen und mit dem Whitebox-Modell simulierten Spannung dargestellt. Dabei werden der Scheitelwert und die Frequenz der Spannung unterschätzt. Dies wird insbesondere am Sternpunkt deutlich.

Gemessene und berechnete Scheitelwerte der Spannungen in zwei Strängen sind in Abb. 15 dargestellt. Da es sich um eine symmetrische Wicklung handelt, sind die gemessenen und simulierten 
Tab. 4. Bezeichnungen der Größen des Greybox-Modells

\begin{tabular}{ll}
\hline Bezeichnung & Bedeutung \\
\hline$\underline{U}_{\text {in }}(x, t)$ & Eingangsspannung bei ausgeprägter Frequenz \\
$\tilde{U}(x, t)$ & Ortsabhängige Spannung im Zeitbereich \\
$x$ & Um Sprung reduzierte Spannung im Zeitbereich \\
$x^{*}$ & Position \\
$\omega$ & Festgelegter Ort \\
$\omega^{*}$ & Kreisfrequenz \\
$d$ & Festgelegte Kreisfrequenz, dominante Frequenz \\
$U(d, t)=U(0,1)$ & Länge der Wicklung \\
$U(d / 2, t)$ & Klemmspannung \\
\hline & Sternpunktspannung \\
\hline
\end{tabular}

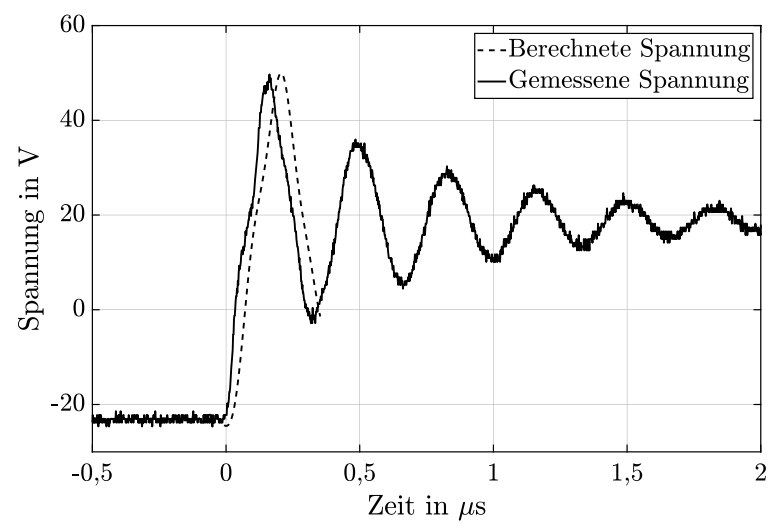

(a) Gemessene und modellierte Spannung in der ersten Windung (von den Klemmen aus betrachtet).

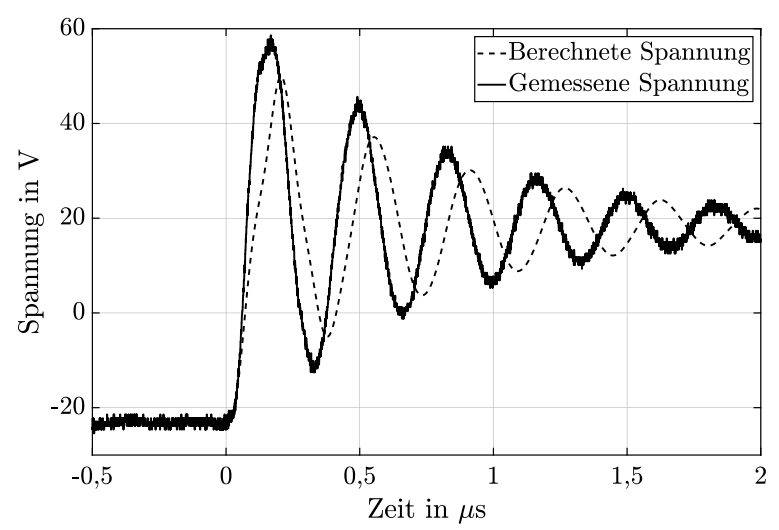

(b) Gemessene und modellierte Spannung am Sternpunkt.

Abb. 14. Mess- und Simulationsergebnisse des Lumped-ParameterModells

Spannungen nahezu symmetrisch zum Sternpunkt. Der Scheitelwert wird für jede Windung unterschätzt. Die Differenz zwischen simulierter und gemessener Spannung nimmt mit der Nähe zum Sternpunkt zu.

\subsection{Analyse im Frequenzbereich}

Gemessene und modellierte Spannung stimmen beim GreyboxModell am Sternpunkt beziehungsweise an den Klemmen miteinander überein. Die Abweichung zwischen gemessener und simulierter Spannung ist darum bei Windungen, die in unmittelbarer Nähe zum Sternpunkt oder zu den Klemmen liegen besonders gering

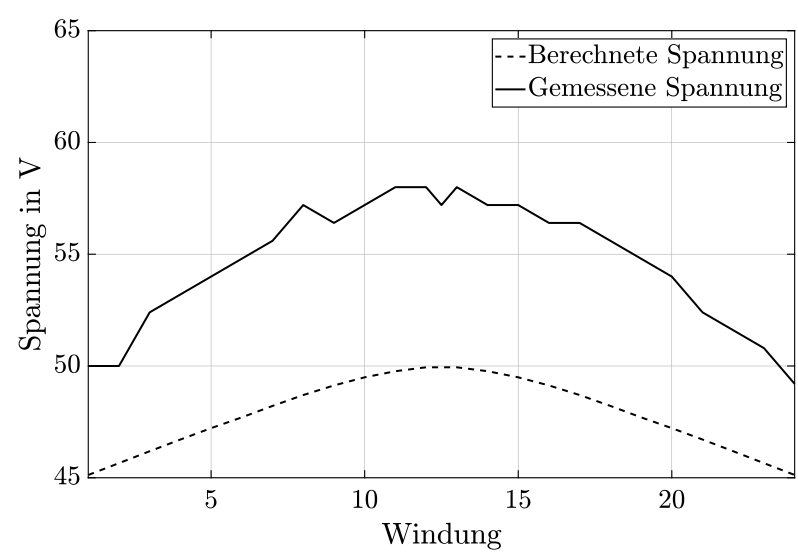

Abb. 15. Gemessene und mit dem Whitebox-Modell berechnete Amplitude der Spannung

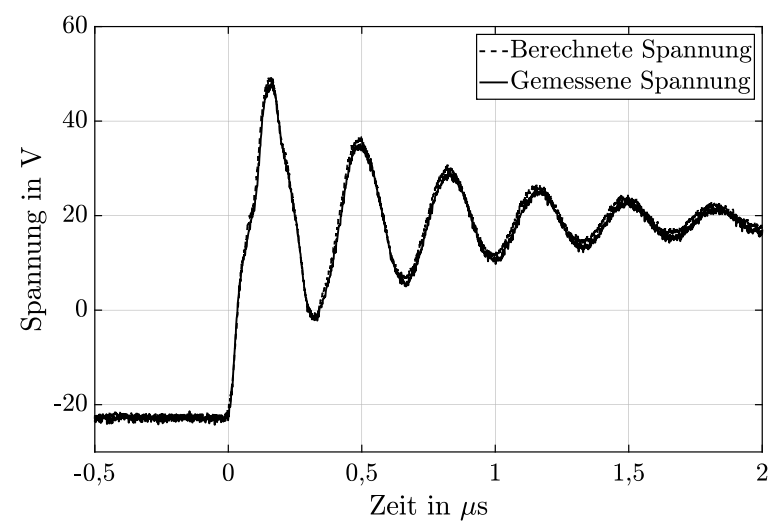

(a) Gemessene und modellierte Spannung in der ersten Windung (von den Klemmen aus betrachtet).

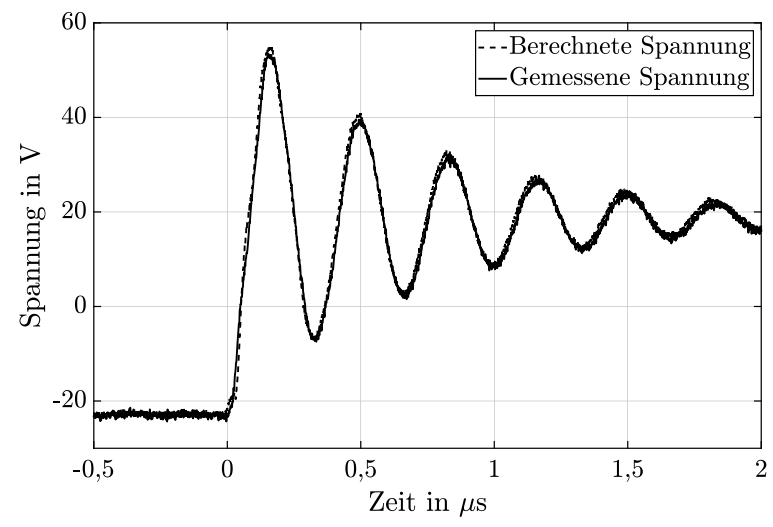

(b) Gemessene und modellierte Spannung in Windung 21.

Abb. 16. Mess und Simulationsergebnisse der Modellierung im Frequenzbereich

(vgl. Abb. 16(a)). Die größte Abweichung zwischen Messung und Simulation tritt in Windung 21 auf (vgl. Abb. 16(b)). Insbesondere die Frequenz der angeregten Schwingung und die Spannungsanstiegsgeschwindigkeit $\mathrm{d} u / \mathrm{d} t$ werden deutlich genauer abgebildet als vom Whitebox-Modell.

In Abb. 17 sind gemessener und berechneter Scheitelwert der Spannung für jede Windung entlang der Wicklung abgebildet. Die 


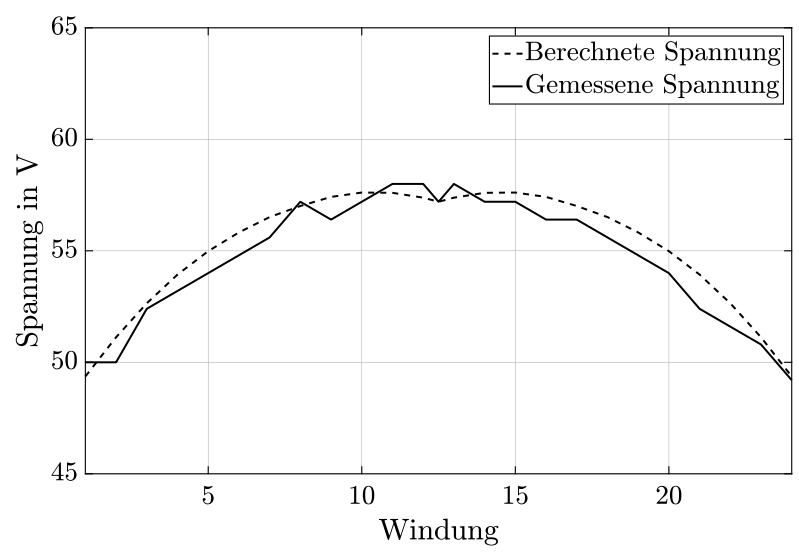

Abb. 17. Gemessene und mit dem Greybox-Modell berechnete Amplitude der Phase-Erde Spannung

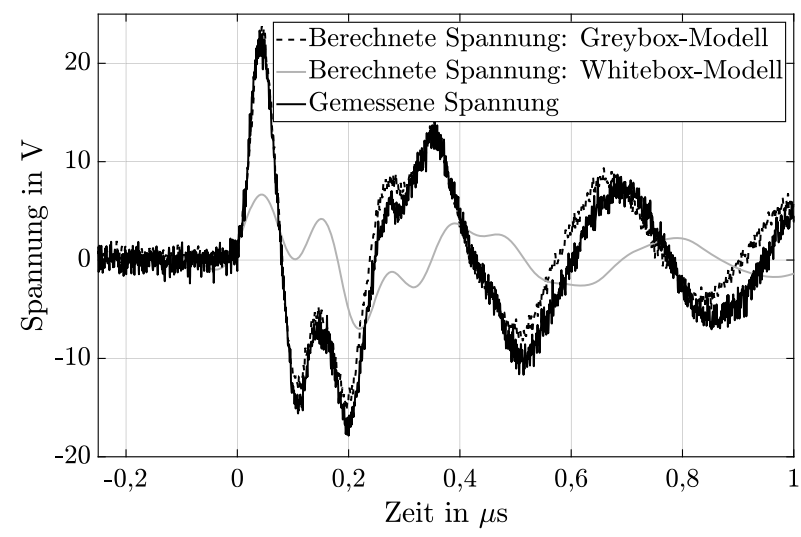

Abb. 18. Spannungsfall über der Windungsisolierung

Differenz zwischen gemessener und berechneter Maximalspannung ist in der Mitte des jeweiligen Stranges am größten.

\subsection{Spannungsfall innerhalb einer Nut}

Mit Hilfe der Modellierung der Spannungsverteilung soll vorhergesagt werden, wie groß der Spannungsfall über der Isolierung ist. Studien zum Vergleich der Teilentladungseinsetzspannungen von Windungsisolierung und Nutgrundisolierung zeigen, dass Teilentladungen im Falle der Windungsisolierung schon bei deutlich geringeren Spannungen auftreten [11]. Darum ist es zur Auslegung des Isolierstoffsystems notwendig, den Spannungsfall über der Windungsisolierung vorhersagen zu können. Bei der hier untersuchten elektrischen Maschine liegen Leiter verschiedener Stränge in einer Nut, ohne von einem Phasentrenner separiert zu werden. Der größte auftretende Spannungsfall ist in Abb. 18 abgebildet. Es ist zu erkennen, dass der gemessene Spannungsfall deutlich höher ist, als durch das Whitebox-Modell prädiziert wird. Die Abweichung zwischen gemessenem und durch das Greybox-Modell prädizierten Spannungsfall ist deutlich geringer.

\section{Schlussfolgerung}

In diesem Artikel werden zwei Modelle zur Abbildung des transienten Spannungsverlaufs innerhalb der Statorwicklung untersucht. Das Whitebox-Modell kann bereits in der Entwurfsphase der Maschine erstellt werden, da ausschließlich Geometrie- und Materialdaten zur Parametrierung benötigt werden. Für das Greybox-Modell werden Messdaten von den Maschinenklemmen und dem im Allgemeinen nicht zugänglichen Sternpunkt benötigt. Das GreyboxModell kann die transiente Spannung in der Maschine genauer prädizieren. Der Scheitelwert der Strang-Erde-Spannung wird vom Greybox-Modell mit einem maximalen Fehler von $4 \%$ vorhergesagt, während der maximale Fehler des Whitebox-Modells 15\% beträgt. Das Greybox-Modell bildet auch Spannungsanstiegsgeschwindigkeit $\mathrm{d} u / \mathrm{d} t$ und angeregte Frequenz besser ab. Darum eignet es sich auch dafür, den Spannungsfall über der Windungsisolierung zu prädizieren. Die Qualität des Whitebox-Modells wird im Wesentlich durch die vorhandenen Geometrie- und Materialdaten bestimmt. Insbesondere Daten zur komplexen Permittivität der Isolierung sowie zur Permeabilität und zu den Verlustparametern des weichmagnetischen Materials müssen über einen großen Frequenzbereich verfügbar sein. Die hier vorgestellten Messungen und Modellierungen werden an einem Stator, der von einer H-Brücke gespeist wird, durchgeführt. Bei der Übertragung der Modelle auf einen kompletten Antriebsstrang müssen die höhere Induktivität, die durch den Rotor verursacht wird, sowie das Schaltmuster des Umrichters berücksichtigt werden.

\section{Danksagung}

Open Access funding provided by Projekt DEAL.

Hinweis des Verlags Der Verlag bleibt in Hinblick auf geografische Zuordnungen und Gebietsbezeichnungen in veröffentlichten Karten und Institutsadressen neutral.

Open Access Dieser Artikel wird unter der Creative Commons Namensnennung 4.0 International Lizenz veröffentlicht, welche die Nutzung, Vervielfältigung, Bearbeitung, Verbreitung und Wiedergabe in jeglichem Medium und Format erlaubt, sofern Sie den/die ursprünglichen Autor(en) und die Quelle ordnungsgemäß nennen, einen Link zur Creative Commons Lizenz beifügen und angeben, ob Änderungen vorgenommen wurden. Die in diesem Artikel enthaltenen Bilder und sonstiges Drittmaterial unterliegen ebenfalls der genannten Creative Commons Lizenz, sofern sich aus der Abbildungslegende nichts anderes ergibt. Sofern das betreffende Material nicht unter der genannten Creative Commons Lizenz steht und die betreffende Handlung nicht nach gesetzlichen Vorschriften erlaubt ist, ist für die oben aufgeführten Weiterverwendungen des Materials die Einwilligung des jeweiligen Rechteinhabers einzuholen. Weitere Details zur Lizenz entnehmen Sie bitte der Lizenzinformation auf http://creativecommons.org/licenses/by/4.0/deed.de.

\section{Literatur}

1. Riedel, A., Masuch, M., Weigelt, M., Gläßel, T., Kühl, A., Reinstein, S., Franke, J. (2018): Challenges of the hairpin technology for production techniques. In 21st international conference on electrical machines and systems (ICEMS) Jeju (S. 2471-2476).

2. Glaessel, T., Seefried, J., Franke, J. (2017): Challenges in the manufacturing of hairpin windings and application opportunities of infrared lasers for the contacting process. In 7th international electric drives production conference (EDPC), Würzburg, (S. 1-7).

3. Pauli, F., Yang, L., Schröder, M., Hameyer, K. (2019): Lebensdauerabschätzung von Wicklungsisolierstoffsystemen in SiC-betriebenen elektrischen Niederspannungsmaschinen. E\&l, Elektrotech. Inf.tech., 136(2), 175-183.

4. Ryu, Y., Han, K. J. (2017): Improved transmission line model of the stator winding structure of an $\mathrm{AC}$ motor considering high-frequency conductor and dielectric effects. In IEEE international electric machines and drives conference (IEMDC), Miami, FL (S. 1-6).

5. Ryu, Y., Park, B., Han, K. J. (2015): Estimation of high-frequency parameters of AC machine from transmission line model. IEEE Trans. Magn., 51(3), 8101404. pp. 1-4.

6. De Gersem, H., Muetze, A. (2012): Finite-element supported transmission-line models for calculating high-frequency effects in machine windings. IEEE Trans. Magn., 48(2), 787-790.

7. De Gersem, H., Henze, O., Weiland, T., Binder, A. (2008): Transmission-line modelling of wave propagation effects in machine windings. In 13th international power electronics and motion control conference, Poznan (S. 2385-2392).

8. Magdun, O., Blatt, S., Binder, A. (2003): Calculation of stator winding parameters to predict the voltage distributions in inverter fed AC machines. In 9th IEEE international symposium on diagnostics for electric machines, power electronics and drives (SDEMPED), Valencia, (S. 447-453). 
9. Lier, M. v., Otten, R. (1973): Planarization by transformation. IEEE Trans. Circuit Theory, 20(2), 169-171.

10. Mahdavi, S., Hameyer, K. (2012): High frequency equivalent circuit model of the stator winding in electrical machines. In XXth international conference on electrical machines (ICEM).

\section{Autoren}

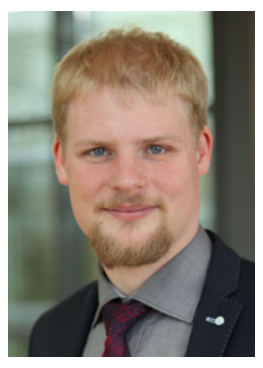

Florian Pauli

studierte Elektrotechnik an der RWTH Aachen und schloss im April 2017 mit dem Master of Science ab. Seitdem arbeitet er als wissenschaftlicher Mitarbeiter am Institut für Elektrische Maschinen der RWTH Aachen. Seine Forschungsschwerpunkte liegen im Bereich der Analyse, Charakterisierung und Auslegung von Isoliersystemen elektrischer Niederspannungsmaschinen.

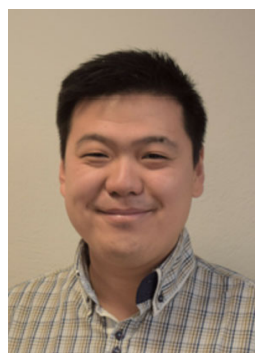

\section{Yixuan Wu}

erwarb seinen Master of Science in Elektrotechnik an der RWTH Aachen und seinen Diplom in Electrical Power Engineering an der KTH Royal Institute of Technology in Stockholm im September 2019. Er war Stipendiat bei der Studienstiftung des deutschen Volkes und arbeitet als Doktorand an der KTH Royal Institute of Technology. Seine Forschungsschwerpunkte liegen im Bereich der Regelung mehrsträngiger elektrischer Maschinen.

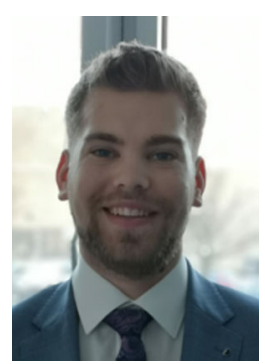

\section{Niklas Driendl}

schloss im Oktober 2018 das Elektrotechnik Studium an der RWTH Aachen mit dem Master of Science ab. Seit Januar 2019 arbeitet er als wissenschaftlicher Mitarbeiter Im Institut für Elektrische Maschinen der RWTH Aachen Seine Forschungsschwerpunkte liegen im Bereich der Auslegung von Isoliersystemen elektrischer Niederspannungsmaschinen und der Untersuchung von Teilentladungsphänomenen.
11. Madonna, V., Giangrande, P., Zhao, W., Buticchi, G., Zhang, H., Gerada, C., Galea, M. (2019): Reliability vs. performances of electrical machines: partial discharges issue. In IEEE workshop on electrical machines design, control and diagnosis (WEMDCD), Athens, Greece (S. 77-82).

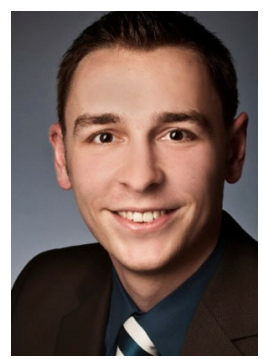

\section{Michael Schröder}

studierte an der RWTH Aachen Elektrotechnik und schloss im Mai 2012 mit dem Diplom ab. Im Anschluss wurde er wissenschaftlicher Mitarbeiter am Institut für Elektrische Maschinen der RWTH Aachen. Von Oktober 2014 bis Oktober 2017 war er Gruppenleiter der Gruppe "Analyse und Design" mit Forschungsthemen im Bereich der weich- und hartmagnetischen Materialien, Isolierstoffsysteme sowie dem strukturdynamischen und akustischen Verhalten elektrischer Maschinen. Seit November 2017 ist er ein Oberingenieur des Instituts. Im Februar 2020 promovierte Dr. Schröder zum Thema "Modellbildung und Analyse von Kraftanregungen in permanentmagneterregten elektrischen Maschinen unter Berücksichtigung von Fertigungseinflüssen".

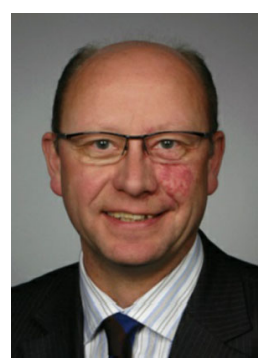

Kay Hameyer

erwarb sein Diplom in Elektrotechnik von der Universität Hannover und promovierte an der Technischen Universität Berlin. Nach seinem Studium arbeitete er für die Robert Bosch GmbH in Stuttgart als Entwicklungsingenieur für permanentmagneterregte Servomotoren und Kraftfahrzeugbordnetzkomponenten. Bis 2004 war Dr. Hameyer Professor für Numerische Feldberechnungen und Elektrische Maschinen an der KU Leuven in Belgien. Seit 2004 ist er Professor und Leiter des Instituts für Elektrische Maschinen der RWTH Aachen. 2006 war er Vizedekan der Fakultät für Elektrotechnik und Informationstechnik der RWTH Aachen und von 2007 bis 2009 Dekan der Fakultät. Seine Forschungsschwerpunkte liegen im Bereich der numerischen Feldberechnung und -optimierung, dem Entwurf und der Regelung elektrischer Maschinen, insbesondere von permanentmagneterregten Maschinen und Drehfeldmaschinen sowie der Entwicklung von Methoden für die virtuelle Realität. Seit vielen Jahren beschäftigt sich die Arbeit von Dr. Hameyer mit der Magnetschwebetechnik für Antriebssysteme, der elektromagnetischen Geräuschanregung elektrischer Maschinen und der Charakterisierung ferromagnetischer Materialien und Isolierwerkstoffe. Er ist Autor von mehr als 350 Journal-Publikationen, mehr als 700 internationalen Konferenzveröffentlichungen und Autor von vier Büchern. Dr. Hameyer ist Mitglied im VDE, IEEE Senior Member und Fellow des IET. 